\title{
Risk of contrast induced nephropathy in the critically ill: a prospective, case matched study
}

Cynthia M Cely ${ }^{1,2}$, Roland MH Schein ${ }^{1,2}$ and Andrew A Quartin ${ }^{1,2^{*}}$

\begin{abstract}
Introduction: Computerized tomography is frequently employed in the critically ill, often using intravenous radiocontrast material. Many of these patients have clinical features that are considered risk factors for contrast induced nephropathy, but are simultaneously at risk for renal injury from other factors related to their acute illnesses. The attributable risk for renal dysfunction from radiocontrast exposure has not been well quantified in this population.

Methods: A prospective matched cohort study was conducted of patients scanned with or without radiocontrast enhancement while receiving intensive care in a Veterans Affairs Medical Center. Patients were matched for prescan measured creatinine clearance, diabetes, mechanical ventilation, and vasopressor use. Measured clearance was followed for three days after scanning. Evolution of nephropathy, as determined by change in measured clearance, was compared within matched pairs.

Results: Fifty-three pairs of patients satisfied matching criteria. Unmatched characteristics were similar among the pairs, including serum creatinine variability during the week preceding scanning ( $67 \pm 85 \%$ among contrast recipients, $63 \pm 62 \%$ among others) and clinical risk factors for renal failure. In 29 pairs, pre-scan measured clearances were less than $60 \mathrm{~mL} /$ minute/1.73 $\mathrm{m}^{2}$. Following scanning, measured clearance declined by at least 33\% in 14 contrast and 19 non-contrast patients (95\% confidence interval for contrast associated difference in nephropathy rates $-27 \%$ to $9 \%$, while a $50 \%$ reduction in clearance persisted three days after scanning in three contrast and nine non-contrast patients (95\% confidence interval for difference in rates $-25 \%$ to $2 \%$ ).

Conclusions: Among established intensive care unit patients declines in glomerular filtration following contrastenhanced scanning are common, but these changes are far more likely to be attributable to factors other than the contrast exposure itself. The upper bound for the incidence of contrast induced renal injury lasting even three days was $2 \%$ in the population studied.
\end{abstract}

\section{Introduction}

Computerized tomography $(\mathrm{CT})$ is invaluable for the management of critically ill patients. While the intravenous administration of iodinated radiographic contrast media (RCM) may be helpful or essential for adequate imaging, the potential complication of contrast induced nephropathy (CIN) is a significant concern. While it has no universally accepted definition, CIN typically refers to an at least modest decline in the glomerular filtration rate (GFR) occurring in the first days following RCM exposure. Its pathophysiology remains uncertain, with

\footnotetext{
* Correspondence: aquartin@med.miami.edu

'Division of Pulmonary, Critical Care, and Sleep Medicine, University of Miami Miller School of Medicine, 1600 NW $10^{\text {th }}$ Ave, Miami, FL, USA 33136 Full list of author information is available at the end of the article
}

proposed mechanisms including medullary hypoxia, free radical generation, and direct tubular toxicity [1].

Depending upon the population studied, CIN incidence has ranged from $1 \%$ to more than $30 \%$ [2-12]. Risk factors include impaired renal function, diabetes, anemia, and hypotension [13-17], all common among ICU patients. Critically ill patients have therefore been presumed to be at relatively high risk for CIN [18-22]. However, analyses of CIN risk factors have focused predominantly on patients undergoing cardiac catheterization and have generally not included unexposed comparator populations. The applicability of these findings to critically ill patients undergoing CT scanning is therefore unknown.

The presumption that critically ill patients are at high risk for CIN has clinical consequences. Perhaps most 
importantly, it may influence how often physicians avoid exposing their critically ill patients to RCM during CT scanning even when it would provide better imaging. Furthermore, when contrast enhancement is utilized a variety of measures may be employed in an attempt to avert CIN, occasionally with unwanted consequences of their own. It is axiomatic that development of rational strategies to prevent CIN, either by prophylaxis or avoidance of RCM exposure altogether, requires accurate estimates of CIN incidence in populations of interest.

CIN is usually recognized by a rise in serum creatinine, with many investigators using as a threshold a $44 \mu \mathrm{mol} / \mathrm{L}$ $(0.5 \mathrm{mg} / \mathrm{dL})$ absolute increase or a $25 \%$ relative increase from before exposure. Identification of CIN among ICU patients using serum creatinine is problematic for several reasons: volatility of filtration with dynamic clinical states; rapidly changing volumes of distribution, from both disease and therapy; and varying creatinine production. These may in part explain disparate reports of CIN incidence among ICU patients $[6,23,24]$. The measured creatinine clearance $\left(\mathrm{mCr}_{\mathrm{Cl}}\right)$, a routinely available test used in trials of critically ill patients [25], intrinsically compensates for these phenomena and may provide better estimates of GFR in the ICU setting [26-28].

As the initial phase of a planned program to investigate the possible utility of very early markers of CIN in the critically ill, we undertook a prospective study of ICU patients requiring CT scanning to better quantify their risk of developing the syndrome and, in particular, to determine whether it could be identified reliably in individual patients. We opted to perform serial measurements of $\mathrm{mCr}_{\mathrm{Cl}}$, before and after scanning, to identify changes in renal function with high accuracy. Because lability of renal function may lead to over-diagnosis of CIN even among less acutely ill patients [29-31], we used matched ICU patients scanned without contrast enhancement as comparators.

\section{Materials and methods}

Subjects were recruited from the medical and surgical ICUs of the Miami Department of Veterans Affairs Medical Center from 12 October 2004 through 2 December 2006. The Human Studies Subcommittee of the Research and Development Committee (the Institutional Review Board of the medical center) approved the study, permitting urine collections before informed consent, but with consent required for sample analysis and inclusion in the study.

CTs were considered evaluable if done while the patient was in the ICU, a urine collection on that day was completed before the scan and the patient was expected to remain in the ICU with an indwelling urinary catheter for the succeeding three days.

\section{Urine samples for creatinine clearance}

Urine was collected nightly from all ICU patients with indwelling urinary catheters who were not receiving renal replacement therapy, with a standing order to start collections at approximately 11:00 PM. Exact start times were recorded on data sheets supplied for the study. Collections were ended the next morning, with the exact completion time also recorded. Urine collection intervals thus included the time of routine serum creatinine sampling for ICUs in our institution. Collected urine volumes were measured with laboratory grade graduated cylinders.

For the few patients transferred out of the ICU within three days of a study CT, urine collections were continued in the same fashion on the ward.

\section{Data collection}

Age, sex, ethnicity, height, weight and history of hypertension, diabetes, liver disease and congestive heart failure were recorded. The last serum albumin and hemoglobin measurements before scanning were documented, along with highest and lowest serum creatinine values from the preceding week. All clinical measurements of serum creatinine and urea nitrogen, along with the times samples were obtained, from 48 hours before to 72 hours after scanning, were logged. Data on exposure to nephrotoxins and drugs that block tubular creatinine secretion (cimetidine and trimethoprim) and administration of agents proposed as prophylaxis against CIN, including sodium bicarbonate and $\mathrm{N}$-acetylcysteine, were collected.

The body area scanned, along with the type and volume of RCM, was noted. Urine output from the three hours and calendar day before scanning, and net fluid balance from the 12 hours and calendar day before scanning, were recorded. Urine outputs and fluid balances from the scan day and the succeeding three days were also recorded. Hemodynamic data included lowest blood pressure during the 24 hours before scanning, hours from last vasopressor use to the time of the scan, and lowest blood pressures and hours of vasopressor use from the three consecutive 24 hour periods following scanning.

\section{Study outcomes}

The principal endpoint, chosen to be very sensitive to even modest effects of RCM exposure, was a 33\% reduction in $\mathrm{mCr}_{\mathrm{Cl}}$ relative to day 0 on any of the three subsequent days. $\mathrm{mCr}_{\mathrm{Cl}}$ was calculated using linear interpolation of serum creatinine measurements to estimate serum creatinine at the midpoint of urine collection periods and was considered $0 \mathrm{~mL} /$ minute in the event of death.

Secondary endpoints included a $50 \%$ reduction in $\mathrm{mCr}_{\mathrm{Cl}}$ at any time, a $33 \%$ reduction in $\mathrm{mCr}_{\mathrm{Cl}}$ at three days after scanning and, most relevant clinically, a 50\% reduction in $\mathrm{mCr}_{\mathrm{Cl}}$ persisting three days after scanning. Deterioration according to the Acute Kidney Injury 
(AKI) Network classification of renal disease [32], formulated after this investigation was initiated, was later added as an endpoint. (AKI Network criteria for oliguria were only considered satisfied if present when averaged over full days, rather than the six or 12 hour periods permitted by the Network guidelines, because of the pre-existing data format.) Relative changes in CockcroftGault (CG) [33] and six variable Modified Diet in Renal Disease (MDRD) [34] estimated GFR were also determined.

\section{Analysis}

Patients who underwent a CT with RCM were paired with a patient scanned without RCM if the following could be matched: day $0 \mathrm{mCr}_{\mathrm{Cl}}$, adjusted for body surface area, within $10 \%$; vasopressor dependency within four hours preceding the scan; requirement for invasive ventilatory support at the time of scanning; and history of diabetes mellitus. Additional restrictions imposed by the computerized matching algorithm included: scans were only eligible if patients did not have other RCM exposures from seven days before until three days after the scan; and patients could contribute only one matched scan.

Differences in dichotomous variables between matched RCM and non-RCM patients were evaluated using McNemar's test with the binomial distribution, with confidence intervals (CIs) calculated using Newcombe's modification of the Wilson score interval [35]. The significance of differences in continuous variables was evaluated using the paired $t$ test or Wilcoxon's test, depending upon the distribution of differences within pairs. Analyses were done using NCSS 2004 (Kaysville, UT, USA) and PASW Statistics 17.0 (SPSS, Chicago, IL, USA).

\section{Results}

\section{Pre-scan data}

Throughout the study period 727 CT scans, 173 using RCM, were performed during 2,228 ICU admissions. Two patients with otherwise evaluable scans declined to participate, leaving 185 patients with 299 evaluable scans. A total of 73 patients received RCM for 79 evaluable scans, and matches were found for 53 of these patients (Figure 1). Matched and unmatched pre-scan profiles are provided in Table 1. Among matching criteria, invasive ventilation was employed for $22(42 \%)$ pairs, and vasopressors within the four hours preceding scanning in two $(4 \%)$ pairs. Fifteen (28\%) pairs had diabetes mellitus. The measured creatinine clearances were $66 \pm 30 \mathrm{~mL} /$ minute $/ 1.73 \mathrm{~m}^{2}$ (mean $\pm \mathrm{SD}$ ) and $65 \pm 29 \mathrm{~mL} /$ minute $/ 1.73 \mathrm{~m}^{2}$ among the RCM and non-RCM patients respectively.

Typical of Department of Veterans Affairs populations, 105 (99\%) of the patients were men. RCM and non-RCM patients were similar with respect to disorders that have been associated with CIN, including liver disease and congestive heart failure (current or New York Heart Association class III or IV by history), present in $30(28 \%)$ and $14(13 \%)$ patients respectively (Table 2). Both groups had marked relative hypotension within the 24 hours preceding scanning (mean arterial pressure $26 \pm 21 \mathrm{~mm} \mathrm{Hg}$ below pre-illness baseline in RCM patients and $28 \pm 21 \mathrm{~mm} \mathrm{Hg}$ in non- $\mathrm{RCM}$ patients, $P>0.6)$, with $38(36 \%)$ patients having mean arterial pressures less than $60 \mathrm{~mm} \mathrm{Hg}(P>0.4$ for difference between groups). Eight (15\%) patients in each group had been newly diagnosed with sepsis during the 48 hours preceding scanning. Fluid balances over the 12 hours preceding scanning were similar among patients who received RCM and those who did not $(1,132 \pm$ $1,550 \mathrm{~mL}$ versus $1,296 \pm 2,159 \mathrm{~mL}, P=0.50$ ) (Table 3 ).

The volume of RCM administered was $144 \pm 17 \mathrm{~mL}$, with 31 patients receiving iopromide $(610 \mathrm{mOsm} / \mathrm{L})$ and 22 iodixanol $(290 \mathrm{mOsm} / \mathrm{L})$. Patients receiving $\mathrm{RCM}$ were more likely to have scans of the abdomen/pelvis or chest and less likely of the head $(P<0.02$ for each). CT angiography was performed during 22 scans.

Pairs were well matched with regard to pre-scan renal function (Table 4). Estimated clearances, serum creatinine and urea nitrogen, and urine flows differed little within pairs. Substantial but similar variability of serum creatinine, expressed as percent increase from the observed minimum, was seen in the week preceding scanning in both groups (67 $\pm 85 \%$ among RCM patients, $63 \pm 62 \%$ among non-RCM patients, $P=0.9$ ) (Figure 2). Serum creatinine was generally at or near its pre-scan minimum at the time of scanning.

$\mathrm{N}$-acetylcysteine was given to 31 (58\%) RCM patients and bicarbonate to 33 (62\%), with 42 (79\%) patients receiving some form of putative prophylaxis. Average fluid administration exceeded three liters on the day of scanning among both contrast and non-contrast patients.

\section{Serial measures of renal function}

Clearance measurements were based on urine collections times of $9.41 \pm 1.94$ hours. Accompanying serum creatinine measurements were made 1.3 (IQR 1.0 to 1.5 ) times per study day. The principal outcome, a $33 \%$ decline in $\mathrm{mCr}_{\mathrm{Cl}}$ at any time during the three days following scanning, occurred in 14 (26\%) RCM patients and 19 (36\%) non-RCM patients (95\% CI for RCM associated incidence change $-27 \%$ to $9 \%, P=0.32$ ) (Table 5 , Figure 3 ). $\mathrm{mCr}_{\mathrm{Cl}}$ at three days after scanning was less than half the pre-scan clearance in three (6\%) RCM patients and nine (17\%) nonRCM patients $(95 \% \mathrm{CI}$ for difference $-25 \%$ to $2 \%, P=0.08)$ (Figure 4). The average peak decline in $\mathrm{mCr}_{\mathrm{Cl}}$ was $19 \pm$ $29 \%$ among RCM patients and $26 \pm 39 \%$ among nonRCM patients $(P=0.23)$. N-acetylcysteine and bicarbonate were not associated with superior renal outcome among RCM patients $(P=1.0)$. 


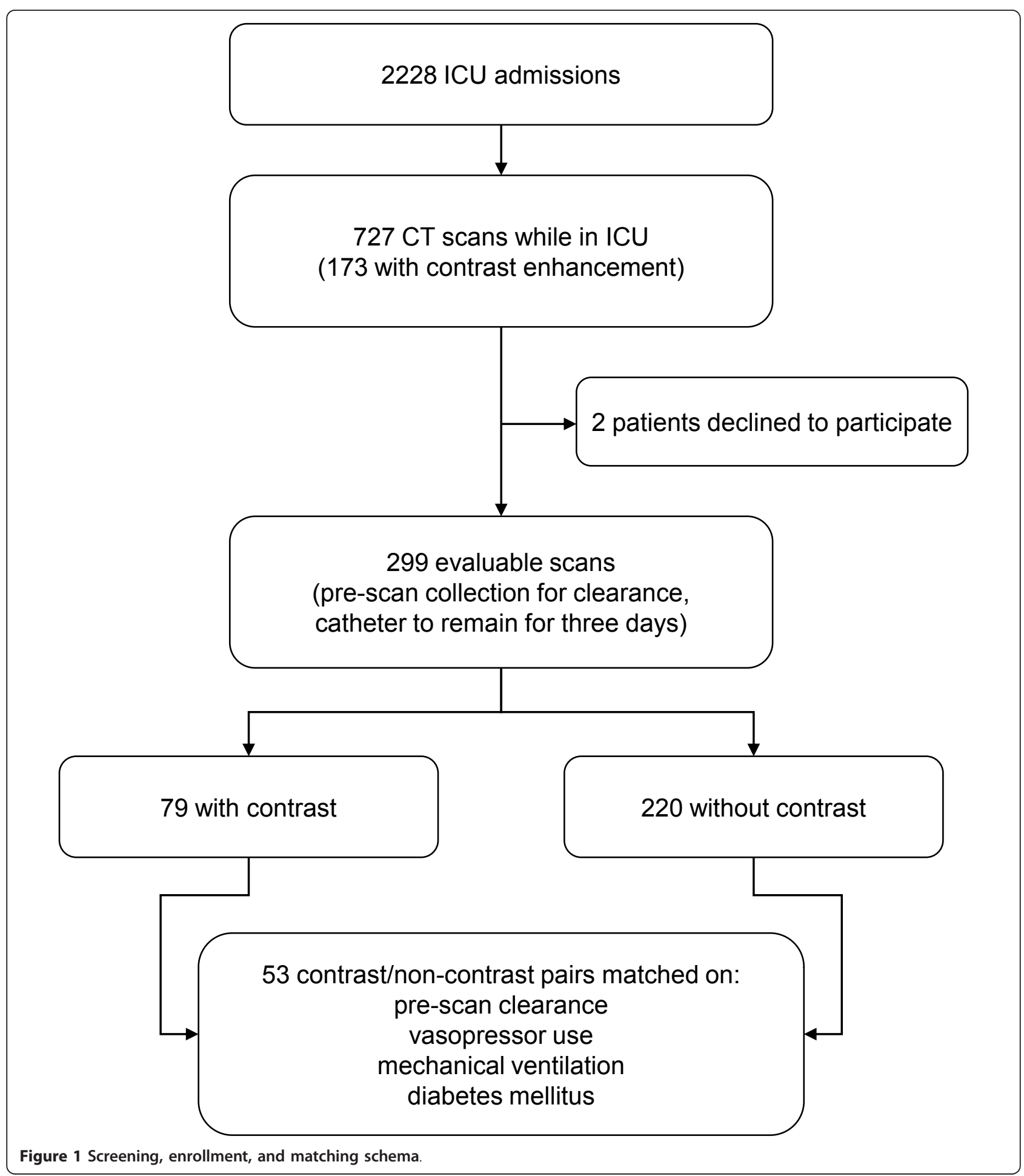

Estimates of GFR not utilizing urine creatinine were less sensitive than $\mathrm{mCr}_{\mathrm{Cl}}$. The peak relative increase in serum creatinine within three days of scanning was $9 \pm$ $19 \%$ among patients exposed to RCM and $16 \pm 58 \%$ among those not $(P=0.38)$. Peak decline in CG estimated clearance was $6 \pm 16 \%$ and $5 \pm 17 \%(P=0.26)$ among RCM and non-RCM patients, respectively, comparable to the values obtained using the MDRD equation $(7 \pm 20 \%$ and $6 \pm 24 \%, P=0.33)$. CG estimates identified ten patients (two who received RCM) and MDRD estimates 11 patients (four who received RCM), as having GFR fall by at least 33\%. 
Table 1 Characteristics of patients at the time scans were performed.

\begin{tabular}{|c|c|c|c|c|}
\hline & $\begin{array}{c}\text { Matched } \\
\text { Contrast } \\
\text { Scans } \\
(n=53) \\
\end{array}$ & $\begin{array}{c}\text { Matched } \\
\text { Noncontrast } \\
\text { Scans } \\
(n=53) \\
\end{array}$ & $\begin{array}{c}\text { Unmatched } \\
\text { Contrast } \\
\text { Scans } \\
(n=26) \\
\end{array}$ & $\begin{array}{c}\text { Unmatched } \\
\text { Noncontrast } \\
\text { Scans } \\
(n=167) \\
\end{array}$ \\
\hline \multicolumn{5}{|l|}{ Matching criteria } \\
\hline $\mathrm{mCr}_{\mathrm{Cl}}, \mathrm{mL} / \mathrm{min} / 1.73 \mathrm{~m}^{2}$ & $66 \pm 30$ & $65 \pm 29$ & $97 \pm 43$ & $44 \pm 33$ \\
\hline Ventilated at time of scan & $22(42 \%)$ & $22(42 \%)$ & $16(62 \%)$ & $98(59 \%)$ \\
\hline Pressor use in $4 \mathrm{hrs}$ before $\mathrm{CT}$ & $2(4 \%)$ & $2(4 \%)$ & $6(23 \%)$ & $19(11 \%)$ \\
\hline Diabetes & $15(28 \%)$ & $15(28 \%)$ & $4(15 \%)$ & $62(37 \%)$ \\
\hline Age, years & $70 \pm 10$ & $67 \pm 13$ & $61 \pm 14$ & $71 \pm 12$ \\
\hline Liver disease & $17(32 \%)$ & $13(25 \%)$ & $5(19 \%)$ & $31(19 \%)$ \\
\hline CHF (current or Class III/IV) & $5(9 \%)$ & $9(17 \%)$ & $3(12 \%)$ & $25(15 \%)$ \\
\hline \multicolumn{5}{|l|}{$\mathrm{S}_{\mathrm{Cr},}, \mu \mathrm{mol} / \mathrm{L}$} \\
\hline Last value before scanning & $92 \pm 30$ & $95 \pm 41$ & $64 \pm 30$ & $133 \pm 84$ \\
\hline Lowest in the preceding week & $82 \pm 28$ & $85 \pm 40$ & $56 \pm 21$ & $108 \pm 65$ \\
\hline Highest in the preceding week & $137 \pm 80$ & $134 \pm 65$ & $80 \pm 31$ & $171 \pm 114$ \\
\hline Last pre-scan BUN, mmol/L & $7.1 \pm 3.6$ & $8.2 \pm 4.6$ & $4.6 \pm 2.5$ & $11.4 \pm 7.1$ \\
\hline \multicolumn{5}{|l|}{ Mean arterial pressure, $\mathrm{mm} \mathrm{Hg}$} \\
\hline Before acute illness (baseline) & $93 \pm 14$ & $92 \pm 14$ & $95 \pm 13$ & $95 \pm 15$ \\
\hline Lowest 24 hrs pre-scan & $67 \pm 16$ & $63 \pm 16$ & $70 \pm 13$ & $65 \pm 15$ \\
\hline $\mathrm{F}_{1} \mathrm{O}_{2}$ at time of scan, $\%$ & $42 \pm 23$ & $39 \pm 16$ & $52 \pm 30$ & $46 \pm 21$ \\
\hline New sepsis before scan & $8(15 \%)$ & $8(15 \%)$ & $8(31 \%)$ & $22(13 \%)$ \\
\hline \multicolumn{5}{|l|}{ Body area scanned } \\
\hline Head & 19 (36\%) & $32(60 \%)$ & $4(15 \%)$ & $64(38 \%)$ \\
\hline Chest & $35(66 \%)$ & $22(42 \%)$ & $16(62 \%)$ & $96(58 \%)$ \\
\hline Abdomen/pelvis & 27 (51\%) & $10(19 \%)$ & $15(58 \%)$ & $44(26 \%)$ \\
\hline Contrast desirable & & $4(8 \%)$ & & $17(10 \%)$ \\
\hline
\end{tabular}

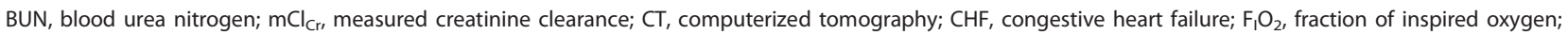
$\mathrm{S}_{\mathrm{Cr}}$, serum creatinine.

Forty four (42\%) patients satisfied AKI Network criteria for acute kidney injury, 23 of whom received RCM and 21 who did not ( $95 \% \mathrm{CI}$ for difference $-14 \%$ to $21 \%, P=0.67$ ). AKI Network level three injury, the most severe gradation, occurred in five (9\%) RCM and 11 (21\%) non-RCM patients $(95 \% \mathrm{CI}$ for difference $-26 \%$ to $4 \%, P=0.21$ ).

In 29 matched pairs both patients had pre-scan $\mathrm{mCr}_{\mathrm{Cl}}$ less than $60 \mathrm{~mL} /$ minute $/ 1.73 \mathrm{~m}^{2}$. Measured clearance after scanning declined by at least 33\% in $5(17 \%)$ patients who received RCM and 12 (41\%) who did not (95\% CI for difference $-46 \%$ to $2 \%, P=0.12$ ). Thirty (52\%) of these patients, half of whom received RCM, qualified as having acute kidney injury by AKI Network criteria. Level three injury occurred in three (10\%) RCM patients and nine (31\%) non-RCM patients ( $95 \%$ CI for difference $-42 \%$ to $3 \%, P=0.15)$.

The investigators judged that RCM enhancement would have been desirable for four of the matched patients (8\%) scanned without it. Only one had a $33 \%$ decline in measured clearance by day three, while none of their four matched contrast-exposed patients had renal injury. Two patients received dialytic support within one week of scanning, one of whom had received RCM. Two patients, neither of whom received RCM, died within 72 hours of scanning.

Matching proved impossible for six of eight patients receiving vasopressors and RCM enhanced scanning. None of these scans were followed by a $33 \%$ decline in measured clearance.

\section{Discussion}

We studied critically ill patients receiving RCM for CT scanning to determine their risk for CIN. Anticipating lability in renal function from critical illness itself, we compared these patients to a matched population scanned without RCM. Significant declines in renal function were frequent, but no more so among those scanned with RCM than those scanned without. It is sobering to note that absent a control population, the declines in renal function observed in the RCM exposed patients would have suggested a high CIN incidence.

To the contrary, CIN appears to have been, at most, rare in the population we studied. Our data suggest that among similar patients fewer than two per 100 should suffer a $50 \%$ loss of filtration persisting even three days as a consequence of RCM use. It is clear from our study 
Table 2 Underlying traits, pre-scan risk factors for CIN and scan characteristics among the $\mathbf{5 3}$ patient pairs.

\begin{tabular}{|c|c|c|c|c|c|}
\hline & $\begin{array}{c}\text { Both } \\
\text { Patients }\end{array}$ & $\begin{array}{l}\text { Neither } \\
\text { Patient }\end{array}$ & $\begin{array}{c}\text { Contrast Patient } \\
\text { Only }\end{array}$ & $\begin{array}{c}\text { Noncontrast } \\
\text { Patient } \\
\text { Only }\end{array}$ & $\begin{array}{c}\text { Contrast Associated Excess } \\
(95 \% \mathrm{Cl})\end{array}$ \\
\hline Diabetes $^{a}$ & $\begin{array}{c}15 \\
28 \%\end{array}$ & $\begin{array}{c}38 \\
72 \%\end{array}$ & $\begin{array}{c}0 \\
0 \%\end{array}$ & $\begin{array}{c}0 \\
0 \%\end{array}$ & \\
\hline Ventilated at time of $\mathrm{CT}^{a}$ & $\begin{array}{c}22 \\
42 \%\end{array}$ & $\begin{array}{c}31 \\
59 \%\end{array}$ & $\begin{array}{c}0 \\
0 \%\end{array}$ & $\begin{array}{c}0 \\
0 \%\end{array}$ & \\
\hline Pressor use in 4 hrs before $\mathrm{CT}^{a}$ & $\begin{array}{c}2 \\
4 \%\end{array}$ & $\begin{array}{c}51 \\
96 \%\end{array}$ & $\begin{array}{c}0 \\
0 \%\end{array}$ & $\begin{array}{c}0 \\
0 \%\end{array}$ & \\
\hline Liver Disease & $\begin{array}{c}4 \\
8 \%\end{array}$ & $\begin{array}{c}27 \\
51 \%\end{array}$ & $\begin{array}{c}13 \\
25 \%\end{array}$ & $\begin{array}{c}9 \\
17 \%\end{array}$ & $-10 \%$ to $24 \%$ \\
\hline CHF (current or Class III/IV) & $\begin{array}{c}2 \\
4 \%\end{array}$ & $\begin{array}{c}41 \\
77 \%\end{array}$ & $\begin{array}{c}3 \\
6 \%\end{array}$ & $\begin{array}{c}7 \\
13 \%\end{array}$ & $-20 \%$ to $5 \%$ \\
\hline Hypertension & $\begin{array}{c}22 \\
42 \%\end{array}$ & $\begin{array}{c}13 \\
25 \%\end{array}$ & $\begin{array}{c}9 \\
17 \%\end{array}$ & $\begin{array}{c}9 \\
17 \%\end{array}$ & $-16 \%$ to $16 \%$ \\
\hline New sepsis before scan & $\begin{array}{c}0 \\
0 \%\end{array}$ & $\begin{array}{l}37 \\
70 \%\end{array}$ & $\begin{array}{c}8 \\
15 \%\end{array}$ & $\begin{array}{c}8 \\
15 \%\end{array}$ & $-15 \%$ to $15 \%$ \\
\hline Nephrotoxin in 3 days pre-scan & $\begin{array}{c}6 \\
11 \%\end{array}$ & $\begin{array}{c}29 \\
55 \%\end{array}$ & $\begin{array}{c}10 \\
19 \%\end{array}$ & $\begin{array}{c}8 \\
15 \%\end{array}$ & $-12 \%$ to $19 \%$ \\
\hline $\begin{array}{l}\text { Trimethoprim/cimetidine pre- } \\
\text { scan }\end{array}$ & $\begin{array}{c}0 \\
0 \%\end{array}$ & $\begin{array}{c}47 \\
89 \%\end{array}$ & $\begin{array}{c}3 \\
6 \%\end{array}$ & $\begin{array}{c}3 \\
6 \%\end{array}$ & $-11 \%$ to $11 \%$ \\
\hline \multicolumn{6}{|l|}{ Body area scanned } \\
\hline Head & $\begin{array}{c}10 \\
19 \%\end{array}$ & $\begin{array}{c}12 \\
23 \%\end{array}$ & $\begin{array}{c}9 \\
17 \%\end{array}$ & $\begin{array}{c}22 \\
42 \%\end{array}$ & $-42 \%$ to $-4 \%$ \\
\hline Chest & $\begin{array}{c}14 \\
26 \%\end{array}$ & $\begin{array}{c}10 \\
19 \%\end{array}$ & $\begin{array}{c}21 \\
40 \%\end{array}$ & $\begin{array}{c}8 \\
15 \%\end{array}$ & $5 \%$ to $42 \%$ \\
\hline Abdomen/pelvis & $\begin{array}{c}7 \\
13 \%\end{array}$ & $\begin{array}{c}23 \\
43 \%\end{array}$ & $\begin{array}{c}20 \\
38 \%\end{array}$ & $\begin{array}{c}3 \\
6 \%\end{array}$ & $15 \%$ to $46 \%$ \\
\hline
\end{tabular}

${ }^{a}$ Matching criterion. CHF, congestive heart failure; CIN, contrast induced nephropathy; CT, computerized tomography.

that CIN occurs infrequently compared to other renal insults in the critically ill and loss of GFR after RCM administration, when it occurs, cannot reliably be ascribed to RCM exposure. The observed magnitude of effect is not a consequence of study size. Even a much larger study of similar patients could only be reasonably anticipated to narrow the confidence bands for significant adverse effects from RCM, but still within the upper or lower bounds of the confidence intervals we found.
Our population had many characteristics considered risk factors for CIN. Most had $\mathrm{mCr}_{\mathrm{Cl}}$ less than $60 \mathrm{~mL} /$ minute $/ 1.73 \mathrm{~m}^{2}$ before scanning. Anemia, diabetes, congestive heart failure and advanced age were common. A widely cited tool [14] for predicting CIN incidence, developed from a population undergoing cardiac catheterization, classified most of our patients as being at moderate or high risk. The predicted incidence was $17 \%$, even without accounting for the relative hypotension and recent

Table 3 Matched patient characteristics before scanning.

\begin{tabular}{|c|c|c|c|c|c|}
\hline & Pairs & Contrast & Non-Contrast & Difference & $\begin{array}{c}\text { Paired } \\
P\end{array}$ \\
\hline Age, years & 53 & $70 \pm 10$ & $67 \pm 13$ & $3 \pm 15$ & 0.1578 \\
\hline \multicolumn{6}{|l|}{ Mean arterial pressure, $\mathrm{mm} \mathrm{Hg}$} \\
\hline Before acute illness (baseline) & 48 & $93 \pm 14$ & $92 \pm 14$ & $1 \pm 20$ & 0.7145 \\
\hline Lowest 24 hours pre-scan & 53 & $67 \pm 16$ & $63 \pm 16$ & $3 \pm 18$ & 0.1935 \\
\hline Drop from baseline & 48 & $26 \pm 21$ & $28 \pm 21$ & $-2 \pm 28$ & 0.6136 \\
\hline $\mathrm{F}_{1} \mathrm{O}_{2}$ at time of scan, $\%$ & 53 & $42 \pm 23$ & $39 \pm 16$ & $3 \pm 25$ & 0.3068 \\
\hline 12 hour pre-scan fluid balance, $\mathrm{mL}$ & 48 & $1132 \pm 1550$ & $1296 \pm 2159$ & $-165 \pm 2535$ & 0.4984 \\
\hline Last pre-scan hemoglobin, g/L & 53 & $95 \pm 16$ & $106 \pm 22$ & $-10 \pm 31$ & 0.0195 \\
\hline Serum albumin, g/L & 53 & $25 \pm 6$ & $27 \pm 7$ & $-2 \pm 9$ & 0.1416 \\
\hline Days in ICU at time of scan & 53 & $6.5 \pm 9.7$ & $5.6 \pm 7.1$ & $0.9 \pm 11.7$ & 0.7533 \\
\hline SOFA score & 53 & $3.9 \pm 2.4$ & $4.2 \pm 2.1$ & $0.3 \pm 3.1$ & 0.5088 \\
\hline
\end{tabular}

$\mathrm{F}_{1} \mathrm{O}_{2}$, fraction of inspired oxygen; SOFA, sequential organ failure assessment score. 
Table 4 Pre-scan renal function parameters.

\begin{tabular}{|c|c|c|c|c|c|}
\hline & Pairs & Contrast & Non-Contrast & Difference & $\begin{array}{c}\text { Paired } \\
P\end{array}$ \\
\hline \multicolumn{6}{|l|}{$\mathrm{mCl}_{\mathrm{Cr}}, \mathrm{mL} /$ minute } \\
\hline Scan day, normalized to $\mathrm{BSA}^{\mathrm{a}}$ & 53 & $66 \pm 30$ & $65 \pm 29$ & $1 \pm 5$ & 0.2934 \\
\hline Scan day & 53 & $78 \pm 39$ & $75 \pm 34$ & $3 \pm 16$ & 0.1768 \\
\hline Day before scan & 24 & $78 \pm 27$ & $75 \pm 28$ & $4 \pm 21$ & 0.4102 \\
\hline \multicolumn{6}{|l|}{ Scan day calculated $\mathrm{Cl}_{\mathrm{Cr}} \mathrm{mL} / \mathrm{min}$} \\
\hline Cockcroft-Gault formula & 53 & $86 \pm 33$ & $90 \pm 46$ & $-5 \pm 48$ & 0.8456 \\
\hline MDRD formula & 53 & $75 \pm 28$ & $76 \pm 34$ & $-1 \pm 31$ & 0.8828 \\
\hline \multicolumn{6}{|l|}{$\mathrm{S}_{\mathrm{Cr},}, \mu \mathrm{mol} / \mathrm{L}$} \\
\hline Last value before scanning & 53 & $92 \pm 30$ & $95 \pm 41$ & $-4 \pm 37$ & 0.4860 \\
\hline Lowest in the preceding week & 53 & $82 \pm 28$ & $85 \pm 40$ & $-3 \pm 37$ & 0.6899 \\
\hline Highest in the preceding week & 53 & $137 \pm 80$ & $134 \pm 65$ & $3 \pm 97$ & 0.9753 \\
\hline $\mathrm{S}_{\mathrm{Cr}}$ variability in pre-scan week, $\%$ & 53 & $67 \pm 85$ & $63 \pm 62$ & $4 \pm 106$ & 0.9049 \\
\hline Last pre-scan BUN, mmol/L & 53 & $7.1 \pm 3.6$ & $8.2 \pm 4.6$ & $-1.1 \pm 5.4$ & 0.2053 \\
\hline Urine vol in 3 hrs before scan, $\mathrm{mL}$ & 53 & $311 \pm 365$ & $237 \pm 168$ & $74 \pm 401$ & 0.3503 \\
\hline
\end{tabular}

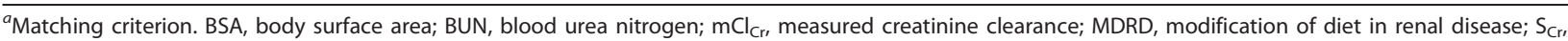
serum creatinine.

renal compromise prevalent among our patients. Indeed, the true incidence of nephropathy within the contrast exposed group was reasonably approximated by the model, but our control group, with similar risks but no contrast exposure, had comparable nephropathy rates.

The only randomized prospective trial of CIN prophylaxis for ICU patients, which compared theophylline with
$\mathrm{N}$-acetylcysteine but employed neither a control arm nor an unexposed arm [8], further underscores the need for caution when ascribing cause. In that study, a $44 \mu \mathrm{mol} / \mathrm{L}$ $(0.5 \mathrm{mg} / \mathrm{dL})$ rise in serum creatinine within 48 hours of imaging was defined as CIN and implicitly attributed to contrast exposure irrespective of comorbidities, and in aggregate occurred after $6 \%$ of scans. We observed a

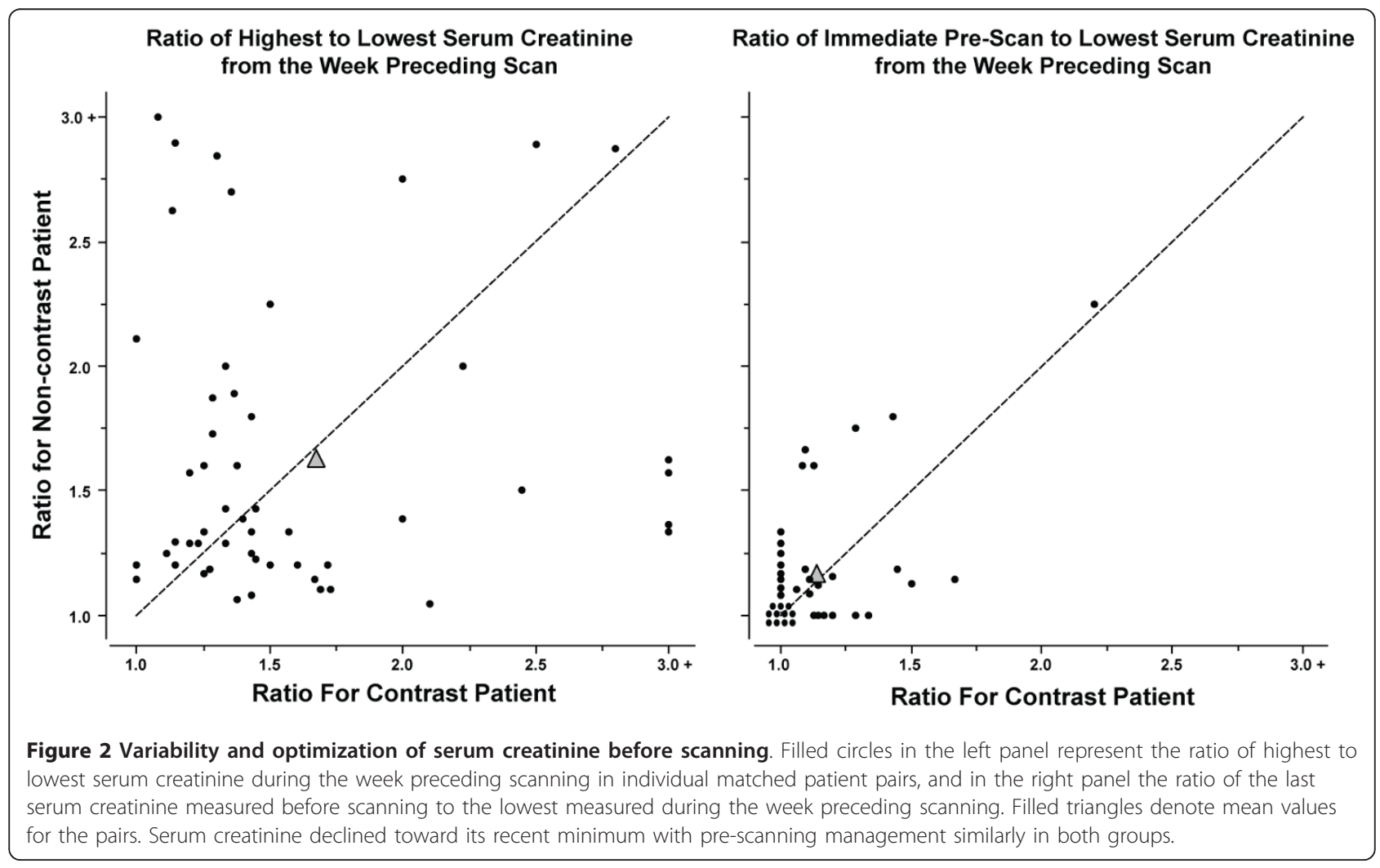


Table 5 Renal dysfunction endpoints after scanning.

\begin{tabular}{|c|c|c|c|c|c|}
\hline & $\begin{array}{c}\text { Both } \\
\text { Patients }\end{array}$ & $\begin{array}{l}\text { Neither } \\
\text { Patient }\end{array}$ & $\begin{array}{c}\text { Contrast Patient } \\
\text { Only }\end{array}$ & $\begin{array}{c}\text { Noncontrast } \\
\text { Patient } \\
\text { Only }\end{array}$ & $\begin{array}{l}\text { Contrast Associated Excess } \\
(95 \% \mathrm{Cl})\end{array}$ \\
\hline $\begin{array}{l}33 \% \text { drop in } \mathrm{mCr}_{\mathrm{Cl}} \text { at any time within } \\
3 \text { days }\end{array}$ & $\begin{array}{c}4 \\
8 \%\end{array}$ & $\begin{array}{c}24 \\
45 \%\end{array}$ & $\begin{array}{c}10 \\
19 \%\end{array}$ & $\begin{array}{c}15 \\
28 \%\end{array}$ & $-27 \%$ to $9 \%$ \\
\hline $33 \%$ drop in $\mathrm{mCr}_{\mathrm{CI}}$ persisting at day 3 & $\begin{array}{c}1 \\
2 \%\end{array}$ & $\begin{array}{c}34 \\
64 \%\end{array}$ & $\begin{array}{c}5 \\
9 \%\end{array}$ & $\begin{array}{c}13 \\
25 \%\end{array}$ & $-30 \%$ to $1 \%$ \\
\hline $\begin{array}{l}50 \% \text { drop in } \mathrm{mCr}_{\mathrm{Cl}} \text { at any time within } \\
3 \text { days }\end{array}$ & $\begin{array}{c}1 \\
2 \%\end{array}$ & $\begin{array}{c}33 \\
62 \%\end{array}$ & $\begin{array}{c}7 \\
13 \%\end{array}$ & $\begin{array}{c}12 \\
23 \%\end{array}$ & $-25 \%$ to $7 \%$ \\
\hline $50 \%$ drop in $\mathrm{mCr}_{\mathrm{CI}}$ persisting at day 3 & $\begin{array}{c}0 \\
0 \%\end{array}$ & $\begin{array}{c}41 \\
77 \%\end{array}$ & $\begin{array}{c}3 \\
6 \%\end{array}$ & $\begin{array}{c}9 \\
17 \%\end{array}$ & $-25 \%$ to $2 \%$ \\
\hline Required dialysis within 7 days of scan & $\begin{array}{c}0 \\
0 \%\end{array}$ & $\begin{array}{l}51 \\
96 \%\end{array}$ & $\begin{array}{c}1 \\
2 \%\end{array}$ & $\begin{array}{c}1 \\
2 \%\end{array}$ & $-8 \%$ to $8 \%$ \\
\hline $\begin{array}{l}\text { AKI Network injury (any level) within } 3 \\
\text { days }\end{array}$ & $\begin{array}{c}11 \\
21 \%\end{array}$ & $\begin{array}{c}20 \\
38 \%\end{array}$ & $\begin{array}{c}12 \\
23 \%\end{array}$ & $\begin{array}{c}10 \\
19 \%\end{array}$ & $-14 \%$ to $21 \%$ \\
\hline AKI Network stage 3 within 3 days & $\begin{array}{c}0 \\
0 \%\end{array}$ & $\begin{array}{c}37 \\
70 \%\end{array}$ & $\begin{array}{c}5 \\
9 \%\end{array}$ & $\begin{array}{c}11 \\
21 \%\end{array}$ & $-26 \%$ to $4 \%$ \\
\hline
\end{tabular}

AKI, acute kidney injury; $\mathrm{mCl}_{\mathrm{Cr}}$, measured creatinine clearance.

similar overall incidence of nephropathy satisfying this definition, $4 \%$. However, all of the events in our study happened to have occurred in patients scanned without contrast (who thus had an $8 \%$ incidence), a group specifically studied because their risk profiles were comparable to patients who were scanned with contrast.
Unlike other CIN investigations, we used $\mathrm{mCr}_{\mathrm{Cl}}$ as the principal assessment of GFR. Both the CG and MDRD formulas presume normal creatinine generation and a static GFR. Assessments of renal function derived solely from serum creatinine suffer the same shortcomings. Among critically ill patients with potentially abnormal

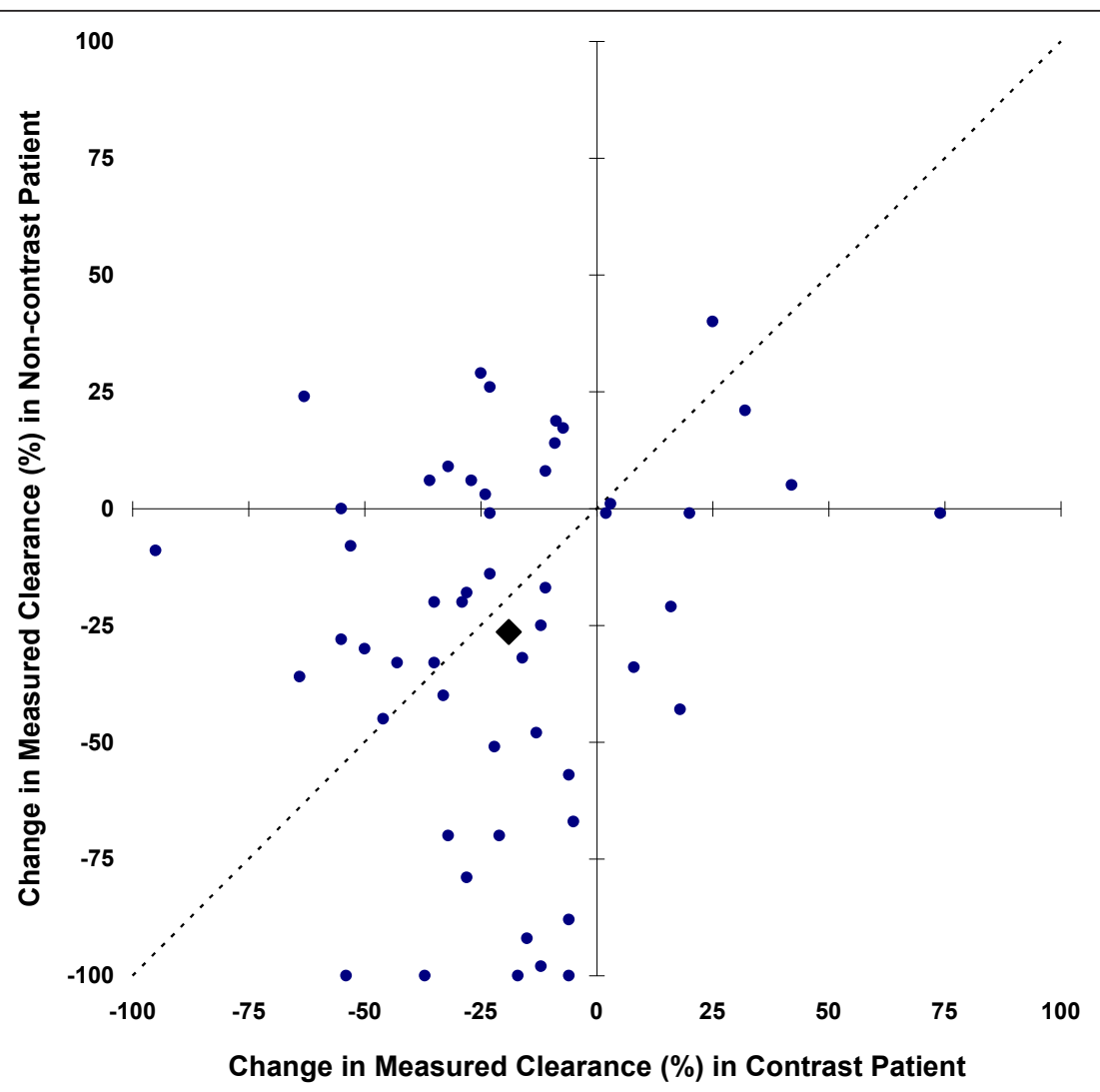

Figure 3 Minimum $\mathrm{mCr}_{\mathrm{Cl}}$ within the three days following scanning relative to pre-scan values. Filled circles represent individual matched patient pairs, and the filled diamond mean values for contrast and non-contrast patients. Points above the diagonal line imply greater loss of renal function in the patient who received contrast, and below the line greater loss in the patient who did not receive contrast. 


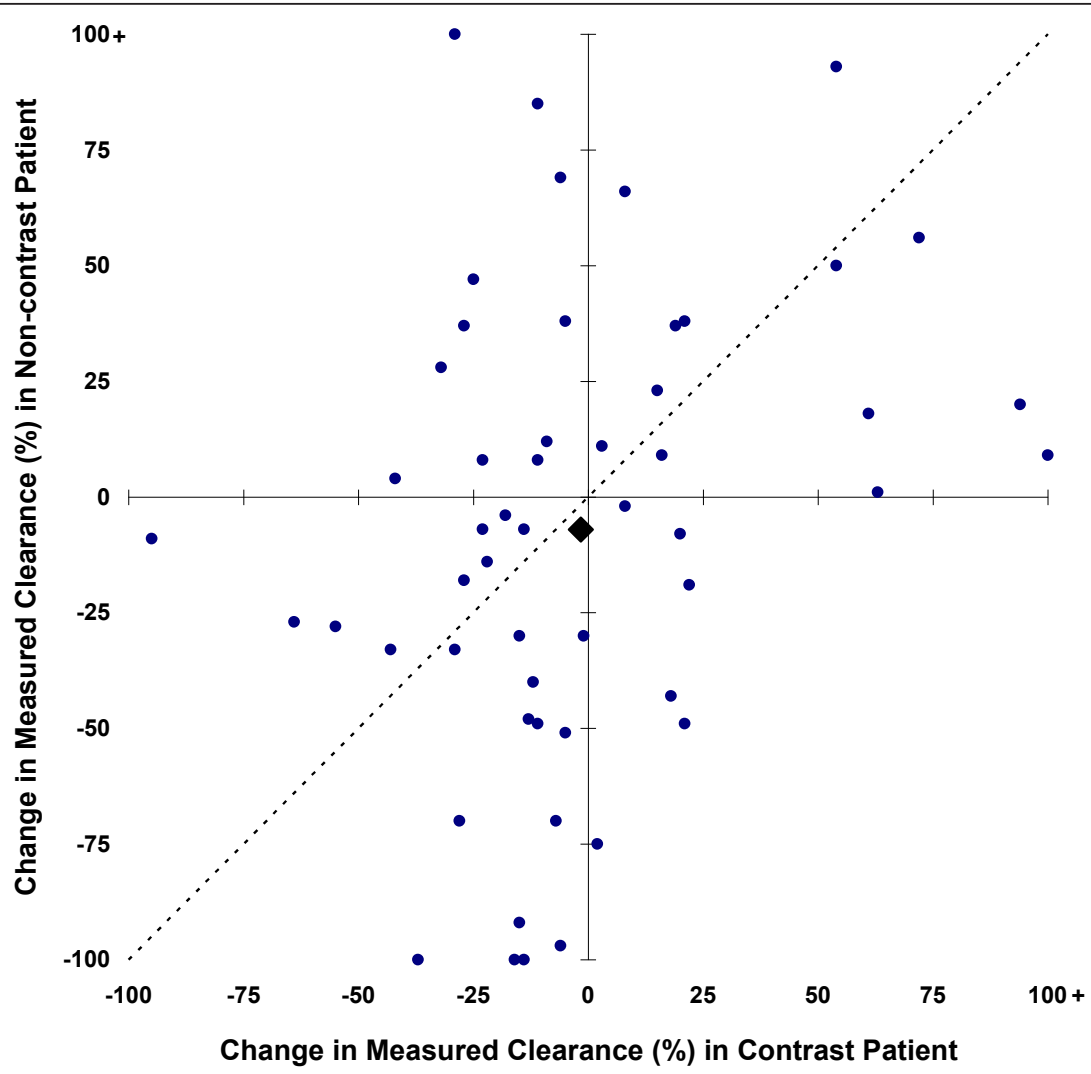

Figure $4 \mathrm{mCr}_{\mathrm{Cl}}$ three days after scanning, relative to pre-scan values. Filled circles represent individual matched patient pairs, and the filled diamond mean values for contrast and non-contrast patients. Points above the diagonal line imply greater loss of renal function in the patient who received contrast, and below the line greater loss in the patient who did not receive contrast.

creatinine production [36,37] and labile filtration, $\mathrm{mCr}_{\mathrm{Cl}}$ may offer advantages over other commonly used estimates of GFR [38].

However, measured creatinine clearance is not without problems. It depends critically upon urine collections being accurately timed and complete. By studying patients with indwelling urinary catheters errors from incomplete voiding were minimized. Accuracy of timing was assured by recording when collections were started and ended, rather than the common practice of specifying the duration of collections in advance.

In addition to changes in GFR, creatinine based measures of renal function are sensitive to fluctuations in the tubular secretion rate of creatinine. Interval institution or cessation of drugs effecting secretion may falsely imply changes in GFR. Use of these drugs, however, was rare, and balanced between RCM and non-RCM patients. Creatinine based measures may underestimate declines in GFR if tubular secretion remains intact, particularly when GFR is initially low. While other techniques for measuring GFR, such as inulin or iothalamate clearance, avoid this shortcoming, they cannot feasibly be employed on a daily basis as this study required.
Properly obtained, $\mathrm{mCr}_{\mathrm{Cl}}$ provides a more accurate picture of GFR in critically ill patients than the serum creatinine or CG or MDRD formulas. However, our key finding remains unchanged irrespective of how GFR is measured, and even what level of dysfunction defines injury: the instability of renal function during critical illness overwhelms any signal from CIN, making it impossible to identify the syndrome accurately. Reinforcing this point, the pre-scanning variability of serum creatinine was of the same magnitude as that required to satisfy CIN definitions of renal injury. Such lability effectively precludes studying interventions to prevent CIN among the critically ill without at least one of the following: a biomarker more specific than GFR for CIN be identified and used as a surrogate endpoint; or very large populations are studied, without attempt to attribute individual events to contrast exposure. This caveat is also applicable to investigations of other potential etiologies of renal injury among the critically ill.

Our study has limitations. While scientifically preferable, a randomized trial of contrast exposure is impracticable, as it would mandate either suboptimal imaging or RCM exposure simply to assess toxicity, violating the 
principal of beneficence. We therefore employed a matched pairs design. Matching might have inadvertently paired RCM patients with unexposed patients at intrinsically higher risk for deterioration of GFR. Comparisons of other unmatched patient characteristics do not suggest this was the case. Furthermore, brain imaging, more common among non-RCM patients, tests for pathologies not generally associated with renal injury.

Because of the stringency of the matching algorithm employed, matching proved impossible for most patients receiving vasopressors. However, no unmatched RCM enhanced scan of a patient who received vasopressors was associated with even a $33 \%$ decline in measured clearance. Somehow including these patients in the analysis could only have diminished further the upper bounds for CIN incidence.

Perhaps more importantly, we could not study patients with RCM exposures shortly after ICU admission, since we required a pre-scan urine collection. These patients may well differ from those scanned later in their ICU stays. For example, there might be salutary effects of the markedly positive pre-scan fluid balances among established ICU patients. We therefore cannot exclude a possibly substantial CIN risk for patients just arriving to the ICU. We also could not study patients whose physicians felt the risk of RCM exposure outweighed the benefits. However, scanning with contrast was judged preferable from an imaging standpoint for fewer than 10\% of patients scanned without contrast, including those who could not be matched.

Previously, one has had to extrapolate from other populations when judging the CIN risk faced by ICU patients and how best to manage it. Cardiac catheterization studies have provided most of these data. The critically ill patients we studied often had findings identified as CIN risk factors in cardiology populations and received RCM volumes comparable to those commonly administered during coronary angiography. However, there are potentially important differences between these populations: critically ill patients received considerably more fluid than recommended for cardiac catheterization CIN prophylaxis; exposure to certain drugs effecting kidney function, including diuretics and angiotensin pathway modulators, is likely higher in the cardiac population; and catheterization simultaneously poses a risk for cholesterol embolization. Our study indeed suggests that extending CIN findings from cardiac catheterization to CT scanning of the critically ill is unwarranted.

\section{Conclusions}

We performed a prospective case matched study to determine the identifiability and frequency of CIN in the critically ill. Our investigation places a very low upper bound on CIN incidence in established ICU patients. The attributable risk for clinically significant renal dysfunction lasting even three days is unlikely to exceed $2 \%$, which should inform planning for imaging studies. Furthermore, in ICU patients attribution of declining filtration to RCM exposure is unreliable because of the background prevalence of deterioration in the absence of exposure. Pending the validation of etiology specific biomarkers, this limitation will likely apply not only to investigations of CIN but also of other potential causes of acute renal injury in the critically ill as well.

\section{Key messages}

- Among established intensive care unit patients, the risk for nephropathy lasting even three days as a consequence of radiocontrast material use appears to be small, less than $2 \%$. This should be considered when planning imaging studies for these patients.

- Individual cases of nephropathy occurring in critically ill patients after use of intravenous radiocontrast material cannot be reliably attributed to the contrast exposure.

- Interventional studies aimed at reducing causespecific renal injury in the critically ill will require either biomarkers more specific than glomerular filtration rate or, alternatively, very large study populations.

\section{Abbreviations}

AKI: acute kidney injury; CG: Cockcroft-Gault; Cl: confidence interval; CIN: contrast induced nephropathy; CT: computerized tomography; GFR: glomerular filtration rate; IQR: interquartile range; $\mathrm{mCr}_{\mathrm{Cl}}$ : measured creatinine clearance; MDRD: Modified Diet in Renal Disease; RCM: radiographic contrast material; SD: standard deviation.

\section{Acknowledgements}

Orlando Gomez-Marin, Ph.D., of the University of Miami Miller School of Medicine, Department of Epidemiology and Public Health, Division of Biostatistics provided statistical consultation.

\section{Author details}

'Division of Pulmonary, Critical Care, and Sleep Medicine, University of Miami Miller School of Medicine, 1600 NW $10^{\text {th }}$ Ave, Miami, FL, USA 33136. ${ }^{2}$ Miami Department of Veterans Affairs Medical Center, 1201 NW $16^{\text {th }}$ St, Miami, Florida, USA 33125.

\section{Authors' contributions}

All authors participated in the study design, data collection, data analysis, and writing of the manuscript. All authors have read and approved the manuscript for publication.

\section{Competing interests}

The authors declare that they have no competing interests.

Received: 5 December 2011 Revised: 24 February 2012 Accepted: 25 April 2012 Published: 25 April 2012

\section{References}

1. Tumlin J, Stacul F, Adam A, Becker CR, Davidson C, Lameire N, McCullough PA: Pathophysiology of contrast-induced nephropathy. Am J Cardiol 2006, 98:14K-20K.

2. Marenzi G, Lauri G, Assanelli E, Campodonico J, De Metrio M, Marana I, Grazi M, Veglia F, Bartorelli AL: Contrast-induced nephropathy in patients 
undergoing primary angioplasty for acute myocardial infarction. $J$ Am Coll Cardiol 2004, 44:1780-1785.

3. Levy EM, Viscoli CM, Horwitz Rl: The effect of acute renal failure on mortality. A cohort analysis. JAMA 1996, 275:1489-1494.

4. Briguori C, Airoldi F, D'Andrea D, Bonizzoni E, Morici N, Focaccio A, Michev I, Montorfano M, Carlino M, Cosgrave J, Ricciardelli B, Colombo A: Renal Insufficiency Following Contrast Media Administration Trial (REMEDIAL): a randomized comparison of 3 preventive strategies. Circulation 2007, 115:1211-1217.

5. Marenzi G, Assanelli E, Marana I, Lauri G, Campodonico J, Grazi M, De Metrio M, Galli S, Fabbiocchi F, Montorsi P, Veglia F, Bartorelli AL: Nacetylcysteine and contrast-induced nephropathy in primary angioplasty. N Engl J Med 2006, 354:2773-2782.

6. Tepel M, van der Giet M, Schwarzfeld C, Laufer U, Liermann D, Zidek W: Prevention of radiographic-contrast-agent-induced reductions in renal function by acetylcysteine. N Engl J Med 2000, 343:180-184.

7. Solomon R, Werner C, Mann D, D'Elia J, Silva P: Effects of saline, mannitol, and furosemide to prevent acute decreases in renal function induced by radiocontrast agents. N Engl J Med 1994, 331:1416-1420.

8. Huber W, Eckel F, Hennig M, Rosenbrock H, Wacker A, Saur D, Sennefelder A, Hennico R, Schenk C, Meining A, Schmelz R, Fritsch R, Weiss W, Hamar P, Heemann U, Schmid RM: Prophylaxis of contrast material-induced nephropathy in patients in intensive care: acetylcysteine, theophylline, or both? A randomized study. Radiology 2006, 239:793-804

9. El-Hajjar M, Bashir I, Khan M, Min J, Torosoff M, DeLago A: Incidence of contrast-induced nephropathy in patients with chronic renal insufficiency undergoing multidetector computed tomographic angiography treated with preventive measures. Am J Cardiol 2008, 102:353-356.

10. Stone GW, McCullough PA, Tumlin JA, Lepor NE, Madyoon H, Murray P, Wang A, Chu AA, Schaer GL, Stevens M, Wilensky RL, O'Neill WW, CONTRAST Investigators: Fenoldopam mesylate for the prevention of contrast-induced nephropathy: a randomized controlled trial. JAMA 2003, 290:2284-2291.

11. Mitchell AM, Kline JA: Contrast nephropathy following computed tomography angiography of the chest for pulmonary embolism in the emergency department. J Thromb Haemost 2007, 5:50-54.

12. Mitchell $A M$, Jones $A E$, Tumlin JA, Kline JA: Incidence of contrast-induced nephropathy after contrast-enhanced computed tomography in the outpatient setting. Clin J Am Soc Nephrol 2010, 5:4-9.

13. Lautin EM, Freeman NJ, Schoenfeld AH, Bakal CW, Haramati N, Friedman AC, Lautin JL, Braha S, Kadish EG, Sprayregen S, Belizon I: Radiocontrast-associated renal dysfunction: incidence and risk factors. AJR 1991, 157:49-58.

14. Mehran R, Aymong ED, Nikolsky E, Lasic Z, lakovou I, Fahy M, Mintz GS, Lansky AJ, Moses JW, Stone GW, Leon MB, Dangas G: A simple risk score for prediction of contrast-induced nephropathy after percutaneous coronary intervention: development and initial validation. J Am Coll Cardiol 2004, 44:1393-1399.

15. Brown JR, DeVries JT, Piper WD, Robb JF, Hearne MJ, Ver Lee PM, Kellet MA, Watkins MW, Ryan TJ, Silver MT, Ross CS, MacKenzie TA, O'Connor GT, Malenka DJ, Northern New England Cardiovascular Disease Study Group: Serious renal dysfunction after percutaneous coronary interventions can be predicted. Am Heart J 2008, 155:260-266

16. Jo S-H, Youn T-J, Koo B-K, Park J-S, Kang H-J, Cho Y-S, Chung W-Y, Joo G-W, Chae I-H, Choi D-J, Oh B-H, Lee M-M, Park Y-B, Kim H-S: Renal toxicity evaluation and comparison between visipaque (iodixanol) and hexabrix (ioxaglate) in patients with renal insufficiency undergoing coronary angiography: the RECOVER study: a randomized controlled trial. J Am Coll Cardiol 2006, 48:924-930

17. Dangas G, lakovou I, Nikolsky E, Aymong ED, Mintz GS, Kipshidze NN, Lansky AJ, Moussa I, Stone GW, Moses JW, Leon MB, Mehran R: Contrastinduced nephropathy after percutaneous coronary interventions in relation to chronic kidney disease and hemodynamic variables. Am J Cardiol 2005, 95:13-19.

18. Bentley ML, Corwin HL, Dasta J: Drug-induced acute kidney injury in the critically ill adult: recognition and prevention strategies. Crit Care Med 2010, 38:S169-174
19. Dennen P, Douglas IS, Anderson R: Acute kidney injury in the intensive care unit: an update and primer for the intensivist. Crit Care Med 2010, 38:261-275.

20. Hock R, Anderson RJ: Prevention of drug-induced nephrotoxicity in the intensive care unit. J Crit Care 1995, 10:33-43.

21. McCullough PA: Acute kidney injury with iodinated contrast. Crit Care Med 2008, 36:S204-211.

22. McCullough PA, Soman SS: Contrast-induced nephropathy. Crit Care Clin 2005, 21:261-280.

23. Haveman JW, Gansevoort RT, Bongaerts AHH, Nijsten MWN: Low incidence of nephropathy in surgical ICU patients receiving intravenous contrast: a retrospective analysis. Intensive Care Med 2006, 32:1199-1205.

24. Polena S, Yang S, Alam R, Gricius J, Gupta JR, Badalova N, Chuang P, Gintautas J, Conetta R: Nephropathy in critically ill patients without preexisting renal disease. Proc West Pharmacol Soc 2005, 48:134-135.

25. VA/NIH Acute Renal Failure Trial Network, Palevsky PM, Zhang JH, O'Connor TZ, Chertow GM, Crowley ST, Choudhury D, Finkel K, Kellum JA, Paganini E, Schein RM, Smith MW, Swanson KM, Thompson BT, Vijayan A, Watnick S, Star RA, Peduzzi P: Intensity of renal support in critically ill patients with acute kidney injury. N Engl J Med 2008, 359:7-20

26. Martin C, Alaya M, Bras J, Saux P, Gouin F: Assessment of creatinine clearance in intensive care patients. Crit Care Med 1990, 18:1224-1226.

27. Hoste EAJ, Damen J, Vanholder RC, Lameire NH, Delanghe JR, Van den Hauwe K, Colardyn FA: Assessment of renal function in recently admitted critically ill patients with normal serum creatinine. Nephrol Dial Transplant 2005, 20:747-753.

28. Villa P, Jimenez M, Soriano M-C, Manzanares J, Casasnovas P: Serum cystatin $\mathrm{C}$ concentration as a marker of acute renal dysfunction in critically ill patients. Crit Care 2005, 9:R139-143.

29. Cramer BC, Parfrey PS, Hutchinson TA, Baran D, Melanson DM, Ethier RE, Seely JF: Renal function following infusion of radiologic contrast material. A prospective controlled study. Arch Intern Med 1985, 145:87-89.

30. Bruce RJ, Djamali A, Shinki K, Michel SJ, Fine JP, Pozniak MA: Background fluctuation of kidney function versus contrast-induced nephrotoxicity. AJR 2009, 192:711-718

31. Heller CA, Knapp J, Halliday J, O'Connell D, Heller RF: Failure to demonstrate contrast nephrotoxicity. Med J Aust 1991, 155:329-332.

32. Mehta RL, Kellum JA, Shah SV, Molitoris BA, Ronco C, Warnock DG, Levin A, Acute Kidney Injury Network: Acute Kidney Injury Network: report of an initiative to improve outcomes in acute kidney injury. Crit Care 2007, 11 R31.

33. Cockcroft DW, Gault MH: Prediction of creatinine clearance from serum creatinine. Nephron 1976, 16:31-41.

34. Levey AS, Bosch JP, Lewis JB, Greene T, Rogers N, Roth D: A more accurate method to estimate glomerular filtration rate from serum creatinine: a new prediction equation. Modification of Diet in Renal Disease Study Group. Ann Intern Med 1999, 130:461-470.

35. Newcombe RG: Improved confidence intervals for the difference between binomial proportions based on paired data. Stat Med 1998, 17:2635-2650.

36. Leblanc M, Garred L, Cardinal J, Pichette V, Nolin L, Ouimet D, Geadah D: Catabolism in critical illness: estimation from urea nitrogen appearance and creatinine production during continuous renal replacement therapy. Am J Kidney Dis 1998, 32:444-453.

37. Poggio ED, Nef PC, Wang X, Greene T, Van Lente F, Dennis WW, Hall PM: Performance of the Cockcroft-Gault and modification of diet in renal disease equations in estimating GFR in ill hospitalized patients. Am J Kidney Dis 2005, 46:242-252.

38. Erley CM, Bader BD, Berger ED, Vochazer A, Jorzik JJ, Dietz K, Risler T: Plasma clearance of iodine contrast media as a measure of glomerular filtration rate in critically ill patients. Crit Care Med 2001, 29:1544-1550.

doi:10.1186/cc11317

Cite this article as: Cely et al:: Risk of contrast induced nephropathy in the critically ill: a prospective, case matched study. Critical Care 2012 16: R67. 